\title{
The tumor-suppressive role of BATF2 in esophageal squamous cell carcinoma
}

\author{
TIANCI HAN ${ }^{1}$, ZHIYONG WANG ${ }^{1}$, YANG YANG ${ }^{3}$, TIANCI SHU ${ }^{3}$, WEINAN LI ${ }^{2}$, DALI LIU ${ }^{2}$, \\ PEIWEN LI ${ }^{1}$, RUIQUN QI ${ }^{3}$, YI REN ${ }^{3}$, LI LI $^{3}$, HONG LIU ${ }^{4}$, SHUGUANG ZHANG ${ }^{1}$ and LIN ZHANG ${ }^{1}$ \\ Departments of ${ }^{1}$ Thoracic Surgery, ${ }^{2}$ Pathology, ${ }^{3}$ Dermatology and ${ }^{4}$ Otolaryngology, \\ The First Affiliated Hospital of China Medical University, Shenyang, Liaoning 110001, P.R. China
}

Received May 19, 2015; Accepted June 17, 2015

DOI: $10.3892 / o r .2015 .4090$

\begin{abstract}
BATF2 has been found to be decreased in a variety of human malignancies, while its clinical significance and functional roles in esophageal squamous cell carcinoma (ESCC) remain unknown. Herein, the aim of this study was to investigate the expression pattern and to explore the potential functions of BATF2 in ESCC tissues and cell lines. BATF2 mRNA and protein expression levels in human tissues and human ESCC cell lines were evaluated by quantitative real-time polymerase chain reaction (qRT-PCR), western blotting (WB) and immunohistochemical (IHC) analyses. BATF2 was upregulated by transfection of the pcDNA3.1-BATF2 plasmid into KYSE-410 cells. MTT and Transwell assays were used to investigate the effects of BATF2 on cellular proliferation and invasion. Survival curves were plotted using Kaplan-Meier plots and log-rank tests. Cox's proportional hazards regression model was used to analyze univariate and multivariate survival. The results showed that, compared to the matched non-tumor tissues from 36 ESCC patients, $80.56 \%$ (29/36) of the tumor tissues presented downregulation of BATF2 by WB analysis $(\mathrm{P}<0.001)$. The results of IHC in 104 patients who underwent surgery for ESCC showed that the expression of BATF2 was closely related to tumor differentiation $(\mathrm{P}=0.023)$ and lymph node metastasis $(\mathrm{P}=0.027)$, while there was no significant correlation with age $(\mathrm{P}=0.574)$, gender $(\mathrm{P}=0.357)$, tumor location $(\mathrm{P}=0.721)$ and TNM stage $(\mathrm{P}=0.126)$ of the patients. Pathological grade $(\mathrm{P}=0.027)$, clinical stage $(\mathrm{P}=0.000)$, lymph node metastasis $(\mathrm{P}=0.002)$ and BATF2 expression $(\mathrm{P}=0.028)$ were identified as independent prognostic factors for overall survival (OS). In the in vitro studies, upregulation of BATF2
\end{abstract}

Correspondence to: Dr Shuguang Zhang or Professor Lin Zhang, Department of Thoracic Surgery, The First Affiliated Hospital of China Medical University, 155 Nanjing North Street, Heping, Shenyang, Liaoning 110001, P.R. China

E-mail: shgzhang@mail.cmu.edu.cn

E-mail: cmu_zhanglin@aliyun.com

Key words: tumor suppressor, BATF2, esophageal squamous cell carcinoma, overexpression, prognostic marker expression significantly inhibited the proliferation and invasive ability of the human ESCC KYSE-410 cells. In conclusion, as a tumor suppressor, BATF2 serves as a prognostic biomarker of ESCC and it may be a potential therapeutic target for ESCC treatment.

\section{Introduction}

Esophageal cancer is one of the most common malignant tumors and has been ranked as the sixth most common cause of cancer-related death worldwide (1). Among the major histologic types of esophageal cancer, squamous cell carcinoma and adenocarcinoma account for more than $90 \%$ of esophageal cancer cases, and the Chinese are particularly prone to develop esophageal squamous cell carcinoma (ESCC) $(2,3)$. In some regions of Northern and Central China, the incidence of ESCC exceeds 100 cases $/ 100,000$ individuals per year (4). Although strategies for the diagnosis and treatment of ESCC have improved, it is still often diagnosed at the advanced stage; thus, many patients lose the chance for tumor resection $(5,6)$. Under this condition, current therapeutic modalities are less effective and patient survival is low (overall 5-year survival of <20\%) (7). Thus, it is urgent to identify novel biomarkers that represent an effective therapeutic target for the disease.

Basic leucine zipper transcription factor, ATF-like 2 (BATF2), also known as suppressor of AP-1, is a member of the BATF subfamily of basic leucine zipper proteins. It is regulated by IFN and has been implicated in cell growth inhibition and apoptosis, and steady-state BATF2 mRNA expression was detected in multiple lineage-specific normal cells, but not in their transformed/tumorigenic counterparts (8). Recently, much evidence has shown that BATF2 may serve as a marker for the diagnosis and prognosis of non-small cell lung cancer, hepatocellular carcinoma and oral tongue squamous cell carcinoma (9-12). However, its role in esophageal squamous cell cancer remains unknown.

The aim of this study was to investigate the expression of BATF2 in ESCC tissues and cell lines and the significance of BATF2 in human ESCC prognosis by clinical investigation and cellular experiments. qRT-PCR and western blot analysis were applied to assess the BATF2 mRNA and protein expression levels of 36 paired ESCC and their adjacent non-tumor tissues. In addition, MTT and Transwell assays were used to 
ascertain the role of BATF2 in proliferation and invasion of the ESCC cell line KYSE-410. Immunohistochemistry (IHC) was performed to determine the clinical relevance of BATF2 in an additional 104 ESCC tissues.

\section{Materials and methods}

Human samples. A total of 36 paired tumor and their corresponding non-tumor tissues were collected from ESCC patients who had undergone surgical resection at the First Affiliated Hospital of China Medical University in 2013. The fresh tissues were immediately frozen in liquid nitrogen and subsequently stored at $-80^{\circ} \mathrm{C}$ until RNA and protein extraction was performed. In order to investigate the correlations between BATF2 and the clinicopathological characteristics and prognosis of ESCC, an additional 104 paraffin-embedded archived ESCC samples (from patients who underwent complete esophageal cancer resection between July, 2008 and April, 2010) and 30 adjacent non-tumor paraffin-embedded tissues were included in this study for IHC. The patients included 92 males and 12 females, aged between 44 and 80 years (median, 63). None of the patients had received radiotherapy or chemotherapy before surgery. The tumor differentiation grades were classified based on World Health Organization (WHO) criteria. Pathological features were classified based on the tumor-node-metastasis (TNM) classification of the American Joint Committee on Cancer (AJCC), 7th edition (13). Pathology was reviewed to confirm histology and tumor grade by two independent pathologists. Clinicopathological information was retrieved from the medical records, including age, gender, tumor location, TNM stage, differentiation, and lymph node metastasis (Table I). The present study was approved by the Specialty Committee on Ethics of Biomedicine Research, The First Affiliated Hospital of China Medical University. The acquisition and use of human tissue in this study complied with the National Regulations on the Use of Clinical Samples in China. Written informed consent was obtained from the patients in advance.

Cell lines and culture. The human esophageal squamous cancer cell lines KYSE-410 and KYSE-150 were purchased from the Beijing Tumor Cell Bank, Chinese Academy of Medical Sciences (Beijing, China); the human esophageal squamous cancer cell line TE-1 and the normal esophageal epithelial cell line HEEC were purchased from the Type Culture Collection of the Chinese Academy of Sciences (Shanghai, China). All four types of cells were grown in Roswell Park Memorial Institute (RPMI)-1640 medium, supplemented with $10 \%$ fetal bovine serum (FBS) (both from Hyclone, USA) and antibiotics (100 U/ml each of penicillin and streptomycin). All cell lines were cultured at $37^{\circ} \mathrm{C}$ in a humidified atmosphere of $5 \% \mathrm{CO}_{2}$. The cells were detached using $0.25 \%$ trypsin with $0.02 \%$ ethylenediaminetetraacetic acid (EDTA).

Transient infection of BATF2 and infection efficiency. The pcDNA3.1-BATF2 plasmid was purchased from Obio Technology (Shanghai, China). Cells were seeded before transfection using RPMI-1640 with $10 \%$ FBS and without antibiotics. Transfection of the pcDNA3.1-BATF2 and
pcDNA3.1 vectors was performed with Lipofectamine ${ }^{\mathrm{TM}} 2000$ according to the manufacturer's instructions, and the untreated cells served as a blank control. The transfected cells were used $48 \mathrm{~h}$ after the transfection. Before subsequent experiments, the mRNA and protein expression levels of BATF2 in the transfected cells were examined by qRT-PCR and western blot analysis, respectively.

Quantitative real-time polymerase chain reaction ( $q R T-P C R)$. Total RNA was isolated using Takara RNAiso reagent (Takara, Shiga, Japan) according to the manufacturer's instructions. Extracted RNA quantity and quality were analyzed by a spectrophotometer (ND1000; Nanodrop, USA). Complementary DNA was synthesized using a PrimeScript RT reagent kit with gDNA Eraser (Takara) using $2 \mu \mathrm{g}$ RNA. SYBR ${ }^{\circledR}$ Premix Ex Taq $^{\text {TM }}$ II (Takara) and Corbet Rotor Gene 3000 thermocycler were used to perform the qRT-PCR reaction. The PCR primers used were as follows: BATF2 sense, 5'-AGACCCCAAGGAGC AACA and antisense, 5'-CAGGGCGAGGTTGTCTTT; and GAPDH (for normalization) sense, 5'-CTCCTCCTGTTCGACA GTCAGC and antisense, 5'-CCCAATACGACCAAATCCGTT. The amplification conditions of the PCR reaction were set as follows: 1 cycle at $95^{\circ} \mathrm{C}$ for $1 \mathrm{~min}$, then 45 cycles for annealing and extension at $95^{\circ} \mathrm{C}$ for $25 \mathrm{sec}$ and $62^{\circ} \mathrm{C}$ for $45 \mathrm{sec}$. qRT-PCR was performed at least three times, and as a negative control, a no-sample control was included. Relative expression levels of BATF2 were normalized by the internal control gene (GAPDH), and data were analyzed by the comparative threshold cycle $\left(2^{-\Delta \Delta \mathrm{CT}}\right)$ method (14).

Western blot assay. Briefly, RIPA lysis buffer supplemented with $1 \%(\mathrm{v} / \mathrm{v})$ protease inhibitor cocktail and PMSF was used to lyse the tissues and cells. Protein concentrations were measured using the BCA method by a protein assay kit (Beyotime, Haimen, China) on a microplate reader (BioTek, Winooski, VT, USA) according to the manufacturer's instructions. Samples ( $40 \mu \mathrm{g} /$ lane of protein) were separated by electrophoresis on $12 \%$ SDS-PAGE, and then transferred to a polyvinylidene difluoride membrane (Millipore, Billerica, MA, USA) in a wet transfer system (Bio-Rad, USA) at $70 \mathrm{~V}$. The membrane was blocked with Tris-buffered saline plus Tween-20 (TBST) containing 5\% non-fat milk for $1 \mathrm{~h}$. The primary rabbit polyclonal antibody anti-BATF2 (sc-130972, 1:1,000 dilution; Santa Cruz Biotechnology, USA) and rabbit monoclonal antibody anti-GAPDH (ab181602, 1:5,000 dilution; Abcam, UK) were used to incubate the membrane overnight at $4^{\circ} \mathrm{C}$. A secondary antibody (goat anti-rabbit, sc-2004; Santa Cruz Biotechnology) was used at a 1:10,000 dilution for $1 \mathrm{~h}$ at room temperature. Immunopositive bands were visualized using enhanced chemiluminescence (ECL buffer; Beyotime Biotechnology, Haimen, China), and band intensities were quantified using Bio-Imaging systems (MF-Chemibis 2.0, DNR; Bio-Imaging Systems Ltd., Israel) and Quantity One software (Bio-Rad).

Immunohistochemistry. Paraffin-embedded biopsy samples were prepared into 4- $\mu \mathrm{m}$ sections. All tissue slides were deparaffinized in xylene, and then rehydrated by graded ethanol solutions. As an antigen retrieval solution, sodium citrate buffer ( $\mathrm{pH}$ 6.0) was used. The specimens were incubated with 
Table I. Association analyses between the expression levels of BATF2 and the clinicopathological characteristics of the ESCC cases.

\begin{tabular}{|c|c|c|c|c|}
\hline \multirow{2}{*}{$\begin{array}{l}\text { Clinicopathological } \\
\text { features }\end{array}$} & \multirow{2}{*}{$\begin{array}{l}\text { No. of } \\
\text { cases }\end{array}$} & \multicolumn{2}{|c|}{ BATF2 } & \multirow[b]{2}{*}{ P-value } \\
\hline & & Low & High & \\
\hline \multicolumn{5}{|l|}{ Age (years) } \\
\hline$\leq 60$ & 44 & 33 & 11 & 0.574 \\
\hline$>60$ & 60 & 42 & 18 & \\
\hline \multicolumn{5}{|l|}{ Gender } \\
\hline Male & 92 & 65 & 27 & 0.357 \\
\hline Female & 12 & 10 & 2 & \\
\hline \multicolumn{5}{|l|}{ Location } \\
\hline $\mathrm{Ut}$ & 24 & 16 & 8 & 0.721 \\
\hline Mt & 31 & 22 & 9 & \\
\hline $\mathrm{Lt}$ & 49 & 37 & 12 & \\
\hline \multicolumn{5}{|l|}{ Pathological grade } \\
\hline Mod/poor & 58 & 47 & 11 & $0.023^{\mathrm{a}}$ \\
\hline Well & 46 & 28 & 18 & \\
\hline \multicolumn{5}{|c|}{ Lymph node metastasis } \\
\hline Negative & 50 & 31 & 19 & $0.027^{\mathrm{a}}$ \\
\hline Positive & 54 & 44 & 10 & \\
\hline \multicolumn{5}{|l|}{ Clinical stage } \\
\hline $\mathrm{I}+\mathrm{II}$ & 52 & 34 & 18 & 0.126 \\
\hline III+IV & 52 & 41 & 11 & \\
\hline
\end{tabular}

Statistical analyses were performed using Pearson's $\chi^{2}$ test; ${ }^{\mathrm{P}}<0.05$ was considered significant. Ut, upper thoracic; Mt, middle thoracic; Lt, lower thoracic; ESCC, esophageal squamous cell carcinoma.

1:100 rabbit polyclonal primary antibody (sc-130972; Santa Cruz Biotechnology) overnight at $4^{\circ} \mathrm{C}$. Following washing with phosphate-buffered saline (PBS), the tissue sections were incubated with an anti-rabbit secondary antibody for $30 \mathrm{~min}$ at $37^{\circ} \mathrm{C}$. Streptavidin peroxidase reagent was applied for $10 \mathrm{~min}$. The tissue sections were incubated with 3,3'-diaminobenzidine (DAB) solution for $3 \mathrm{~min}$. Finally, the sections were counterstained with hematoxylin. The primary antibody was replaced by PBS as a negative control. The expression of BATF2 was determined by two independent pathologists who were blinded to the clinical data. Briefly, staining intensity was graded from 0 to 3 ( 0 for no immunostaining, 1 for light-brown color, 2 for medium-brown color, and 3 for brown color). The percentage of positive staining was scored as follows: 0 points, $\leq 5 \%$; 1 point, $>5-25 \% ; 2$ points, $>25-50 \%$; and 3 points, $>50 \%$ positive cells The final score of BATF2 expression was the product of the percentage score and intensity: - for 0 points, + for $1-2$ points, ++ for 3-5 points, and +++ for 6-9 points. For analysis, BATF2 expression was divided into 'high' (++ and +++$)$ and 'low' (+ and -). Discrepancies were resolved by discussion.

MTT assay. Cell proliferation activity was assessed by MTT assay. Forty-eight hours after transient transfection, the cells $\left(2 \times 10^{3} /\right.$ well) were plated into 96 -well plates in $200 \mu \mathrm{l}$ culture medium and incubated at $37^{\circ} \mathrm{C}$. The culture medium was replaced every 2 days. After 12, 24, 48 and $72 \mathrm{~h}$ of the seeding, MTT solution (20 $\mu \mathrm{l}, 5 \mathrm{mg} / \mathrm{ml}$; Sigma, USA) was added to each well for $4 \mathrm{~h}$ at $37^{\circ} \mathrm{C}$, and dimethyl sulfoxide solution $(150 \mu \mathrm{l} /$ well) was used to dissolve the precipitate. Optical density of the wells was measured at $490 \mathrm{~nm}$ using a microplate reader (BioTek). Independent experiments were performed three times.

Transwell assay. A Transwell system was used to evaluate the cell invasion. A Transwell chamber with a polycarbonate filter membrane (Corning, USA) was placed in a 24-well plate, Matrigel (BD Biosciences, USA) was used to coat the upper surface of the polycarbonic membrane, and the pore size was $8 \mu \mathrm{m}$. KYSE-410 and two group of transfected cells (48 $\mathrm{h}$ after the transfection) were incubated with RPMI-1640 medium but without FBS for $12 \mathrm{~h}$. All the cells were digested to a suspension in serum-free RPMI-1640 medium, and then the cells $\left(5 \times 10^{3} /\right.$ chamber) were added to the Transwell chamber in $200 \mu \mathrm{l} \mathrm{FBS-free} \mathrm{medium.} \mathrm{RPMI-1640} \mathrm{medium} \mathrm{with} 10 \%$ FBS $(500 \mu \mathrm{l})$ was added to the lower chamber, and the cells were cultured in $5 \% \mathrm{CO}_{2}$ at $37^{\circ} \mathrm{C}$. After $24 \mathrm{~h}$ of incubation, the cells in the upper chamber were carefully removed with a cotton swab and the polycarbonic membrane was fixed with 95\% methanol and stained with hematoxylin. Independent experiments were performed three times. The number of transmembrane cells was counted by inverted microscope in 6 randomly selected fields.

Statistical analysis. SPSS 21.0 software (IBM, USA) was used for statistical analysis. A correlation analysis between the expression of BATF2 and clinicopathological factors was carried out using the Chi-square test. Paired Student's t-test was used to compare the statistical significance between groups. Kaplan-Meier method was used to plot survival curves, and the result was compared using the log-rank test. Cox's proportional hazards regression model was used to analyze univariate and multivariate survival. $\mathrm{P}<0.05$ was considered significant.

\section{Results}

Expression of BATF2 protein and $m R N A$ in ESCC and paired adjacent non-tumor tissues. A low expression of BATF2 was noted in the ESCC tissues compared with the matched adjacent non-tumor tissues at both the mRNA and protein levels. The BATF2 mRNA and protein expression in 36 fresh ESCC specimens and their adjacent non-tumor tissues was examined by qRT-PCR and western blot analysis. BATF2 mRNA expression in the 36 ESCC tumor tissues was decreased in 27 (75\%) samples when compared with the adjacent non-tumor tissues $(\mathrm{P}<0.001$, Fig. 1A). As for the protein level of BATF2 in the 36 paired specimens, the expression of BATF2 in the ESCC specimens was decreased in 29 out of 36 $(80.56 \%)$ when compared with the adjacent non-tumor tissues $(\mathrm{P}<0.0001$, Fig. 1B and $\mathrm{C})$.

Expression of BATF2 protein and $M R N A$ in ESCC cell lines. BATF2 exhibited low expression in the ESCC cell lines. qRT-PCR and western blot analysis showed that BATF2 
A

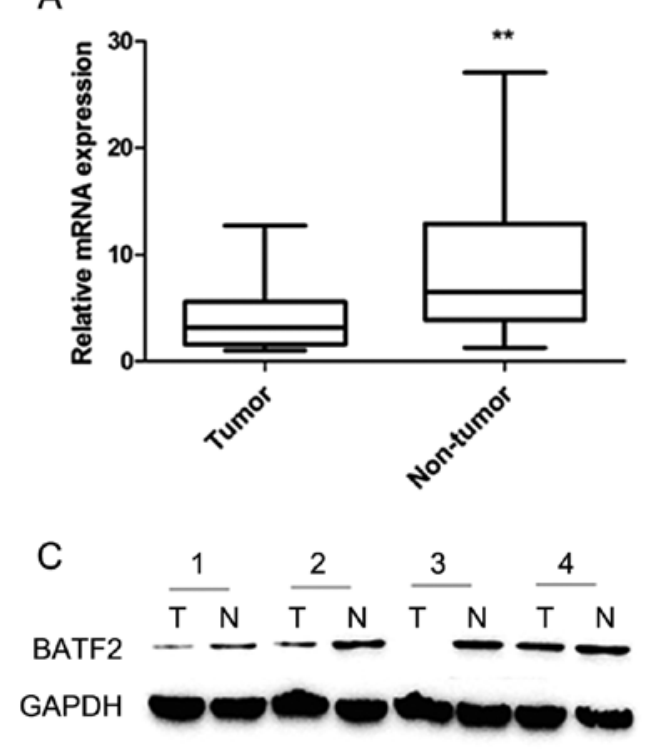

B

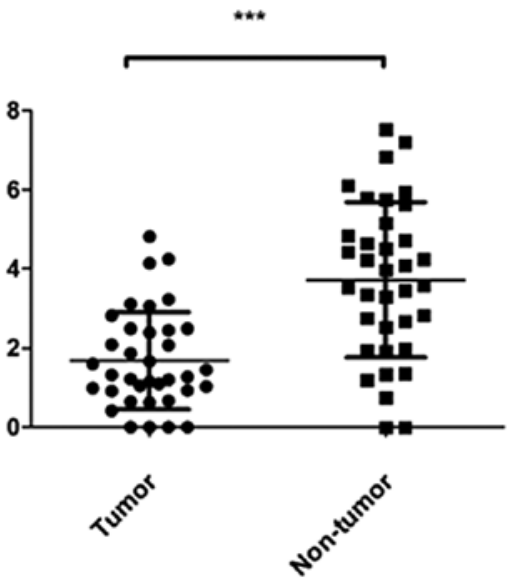

Figure 1. BATF2 expression in ESCC specimens at both the mRNA and protein levels. (A) BATF2 mRNA expression level in ESCC specimens and the adjacent non-tumor tissues. The experiment was replicated three times. ${ }^{* *} \mathrm{P}<0.001$. (B) BATF2 protein expression level in ESCC specimens and the adjacent non-tumor tissues. The experiment was replicated three times. ${ }^{* * *} \mathrm{P}<0.0001$. (C) Representative results of the relative BATF2 expression in 4 paired ESCC tumor (T) and matched adjacent non-tumor $(\mathrm{N})$ tissues by western blot analysis. The experiment was replicated three times. These result suggest that the expression levels of BATF2 mRNA and protein were significantly decreased in the tumor tissues compared with the paired adjacent non-tumor tissues. BATF2, basic leucine zipper transcription factor, ATF-like 2; ESCC, esophageal squamous cell carcinoma.

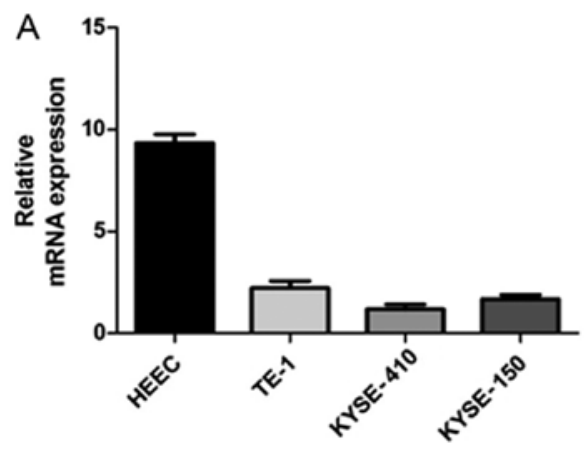

B

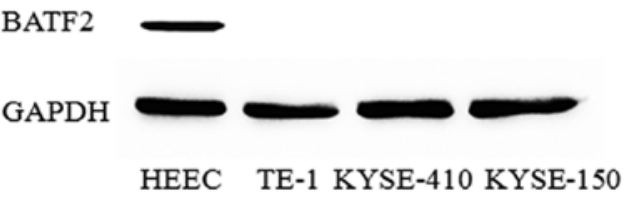

Figure 2. BATF2 expression in ESCC cell lines at both the mRNA and protein levels. (A) qRT-PCR analysis of the relative level of BATF2 mRNA expression in ESCC and normal esophageal epithelial cell lines. Data represent the mean \pm SD of three independent experiments. The experiment was replicated three times. (B) Western blot detection of BATF2 protein expression in ESCC and normal esophageal epithelial cell lines. These data suggest that, consistent with the ESCC tissues, both BATF2 protein and mRNA levels were significantly downregulated in three ESCC cell lines (TE-1, KYSE-410 and KYSE-150) compared with levels in the normal human esophageal epithelium cell line HEEC. The experiment was replicated three times. BATF2, basic leucine zipper transcription factor, ATF-like 2; ESCC, esophageal squamous cell carcinoma.

mRNA (Fig. 2A) and protein expression (Fig. 2B) were significantly decreased in the 3 ESCC cell lines (KYSE-410, KYSE-150 and TE-1) compared with the expression levels in the normal esophageal epithelial cell line HEEC. As shown in Fig. 2B, the highest expression of BATF2 was detected in normal esophageal epithelial HEEC cells, and was barely apparent in the other cell lines.

Role of the overexpression of BATF2 in cellular proliferation and invasion in KYSE-410 cells. To examine the effect of BATF2 expression on cell biological behavior in ESCC, both mRNA and protein of BATF2 were successfully overexpressed in the KYSE-410 cells. qRT-PCR and western blot analysis confirmed that the levels of BATF2 mRNA (Fig. 3A) and protein (Fig. 3B) were significantly upregulated in the KYSE-410 cells transfected with pcDNA3.1-BATF2 when compared with the levels in the cells transfected with the pcDNA3.1 vector or the control group. There was no statistical significance in these levels between the control and vector group ( $\mathrm{P}>0.05)$. To elucidate the role of BATF2 in ESCC proliferation and invasion, three different groups of cells were examined by MTT and Transwell assays. The results revealed that upregulation of BATF2 expression significantly inhibited the proliferation and invasion of the KYSE-410 cells (Fig. 3C-E).

Association between BATF2 protein expression and clinicopathological parameters. We analyzed the association 
A
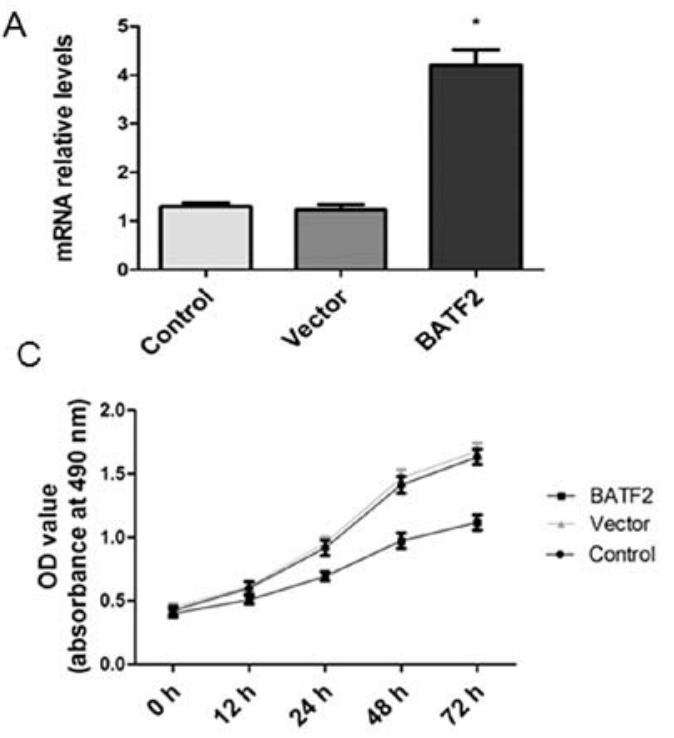

B

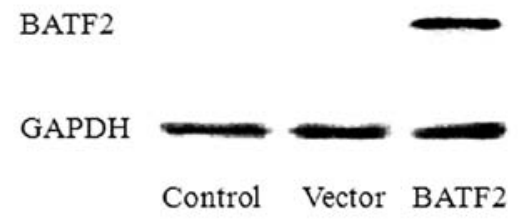

E

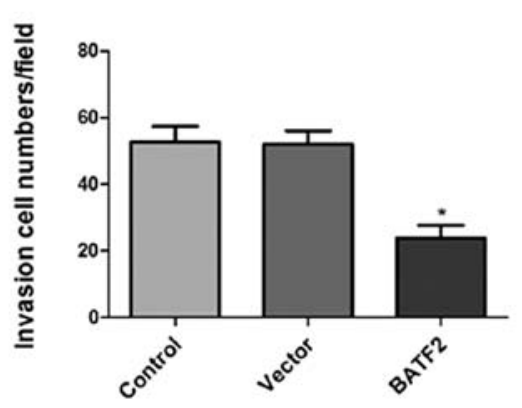

D

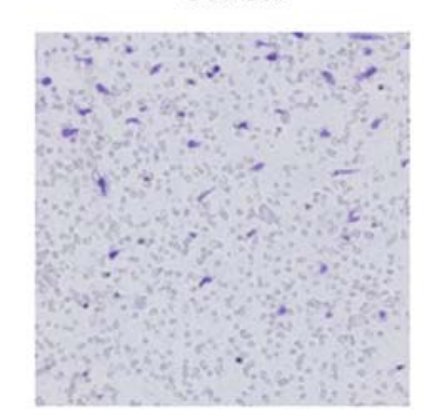

Vector

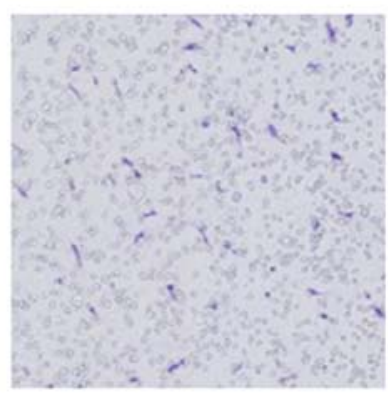

BATF2

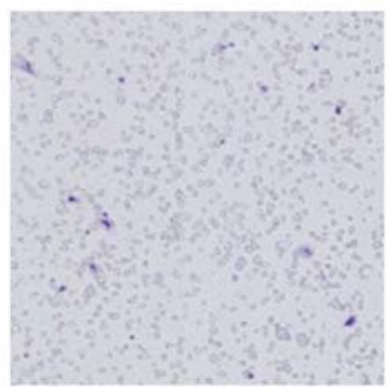

Figure 3. Validation of the efficacy of BATF2 overexpression and the role of BATF2 on cell proliferation and invasion. (A) qRT-PCR analysis of the relative level of BATF2 mRNA in BATF2-overexpressing KYSE-410 cells. The experiment was replicated three times. "P<0.01. (B) Western blot detection of the expression of BATF2 protein in the BATF2-overexpressing KYSE-410 cells. (C) BATF2 overexpression inhibited KYSE-410 cell proliferation. The experiment was replicated three times. (D) Representative images of the KYSE-410 invasion assay after cultivation for $24 \mathrm{~h}$. The experiment was replicated three times. Magnification, x200. (E) Ten random fields (magnification, x200) were selected to count the cells on the lower surface. The result suggest that overexpression of BATF2 inhibited the invasion of KYSE- 410 cells, ${ }^{\prime} \mathrm{P}<0.01$. The values are expressed as the mean \pm SD. The experiment was replicated three times. BATF2, basic leucine zipper transcription factor, ATF-like 2.

between BATF2 protein expression and the clinicopathological features in ESCC (Table I). Based on IHC analysis of the 104 ESCC samples, 75 samples $(72.11 \%)$ had low or no BATF2 expression, and 29 samples $(27.89 \%)$ had moderate or high BATF2 expression. In 30 adjacent non-tumor tissues, 16 cases $(53.33 \%)$ had moderate or high expression of BATF2, and another 14 samples (46.67\%) had low or no expression of BATF2. From the results, it is evident that BATF2 expression was weak or absent in most ESCC tissues, whereas it was moderately or highly expressed in the non-tumor esophageal epithelium (Fig. 4). According to the level of BATF2 expression in the tumor tissues, the 104 ESCC patients were divided into 2 groups, a low and a high expression group. We found that expression of BATF2 was closely related to tumor differentiation $(\mathrm{P}=0.023)$, lymph node metastasis $(\mathrm{P}=0.027)$, while there was no significant correlation with age $(\mathrm{P}=0.574)$, gender $(\mathrm{P}=0.357)$, tumor location $(\mathrm{P}=0.721)$ and TNM stage $(\mathrm{P}=0.126)$ of the patients (Table I).

Prognostic significance of BATF2 expression in ESCC. Among the 104 patients with ESCC, 4 were lost to follow-up and $64(61.54 \%)$ succumbed to death due to primary or recurrent disease. The median duration of follow-up after surgery was 32.1 months (range, 5.4-63.7 months), and patients who died due to postoperative complications were not included in the study. Low expression of BATF2 predicted poor survival of the ESCC patients by Kaplan-Meier analysis ( $\mathrm{P}<0.05$, Fig. 5).

By univariate analysis, the overall survival (OS) of all 104 cases was significantly influenced by the pathological grade, lymph node metastasis, clinical stage and BATF2 expression (Table II). In the multivariate Cox regression analysis, pathological grade $(\mathrm{P}=0.027)$, clinical stage $(\mathrm{P}=0.000)$, lymph node metastasis $(\mathrm{P}=0.002)$ and BATF2 expression $(\mathrm{P}=0.028)$ were identified as independent prognostic factors for OS.

\section{Discussion}

BATF2, belonging to the BATF family (including BATF, BATF2 and BATF3), is a 274-amino acid protein that localizes to the nucleus and contains a basic DNA-binding region and regularly spaced leucine residues known as the leucine zipper 
A

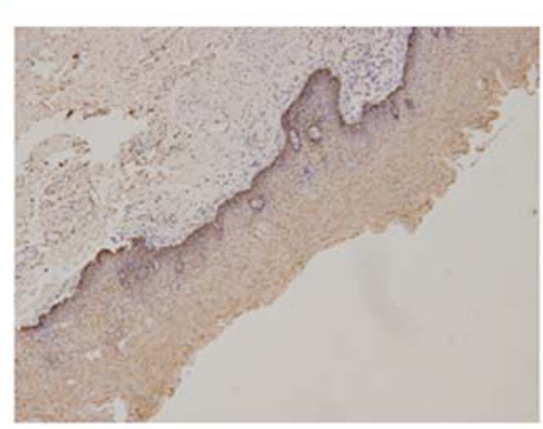

C

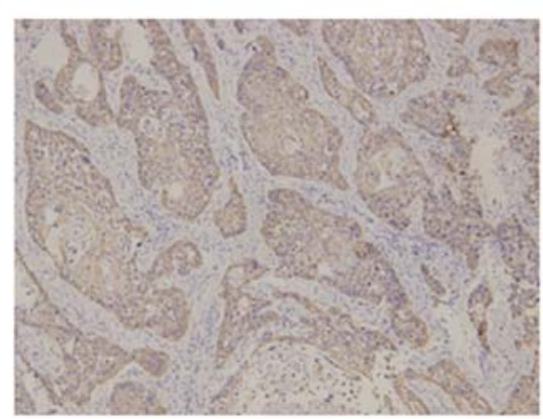

$\mathrm{E}$

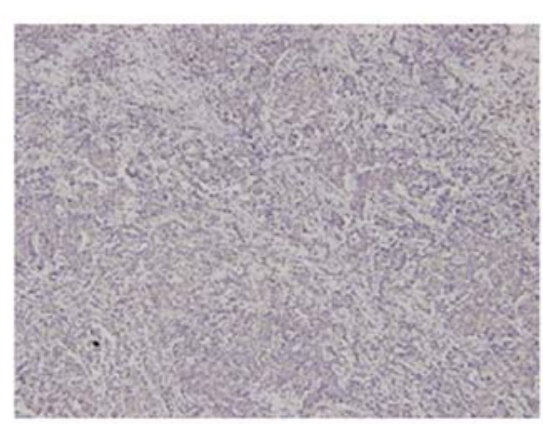

B

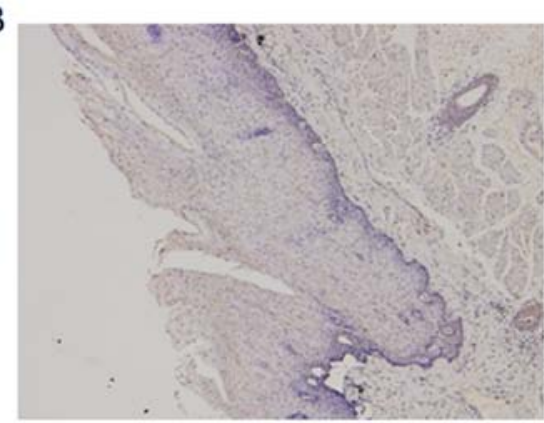

$\mathrm{D}$

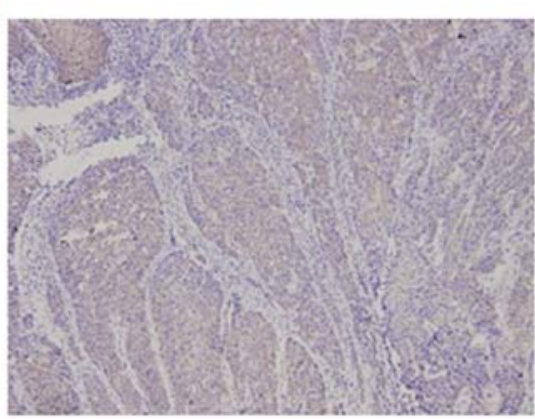

Figure 4. Immunohistochemical analysis of BATF2 expression in esophageal cancer and adjacent non-tumor esophageal tissues. (A) High expression of BATF2 in adjacent non-tumor tissue. (B) No expression of BATF2 in adjacent non-tumor tissue. (C) High staining for BATF2 in well-differentiated ESCC. (D) Moderate staining for BATF2 in well-differentiated ESCC. (E) Weak staining for BATF2 in poorly differentiated ESCC. Magnification, x100. BATF2, basic leucine zipper transcription factor, ATF-like 2; ESCC, esophageal squamous cell carcinoma.

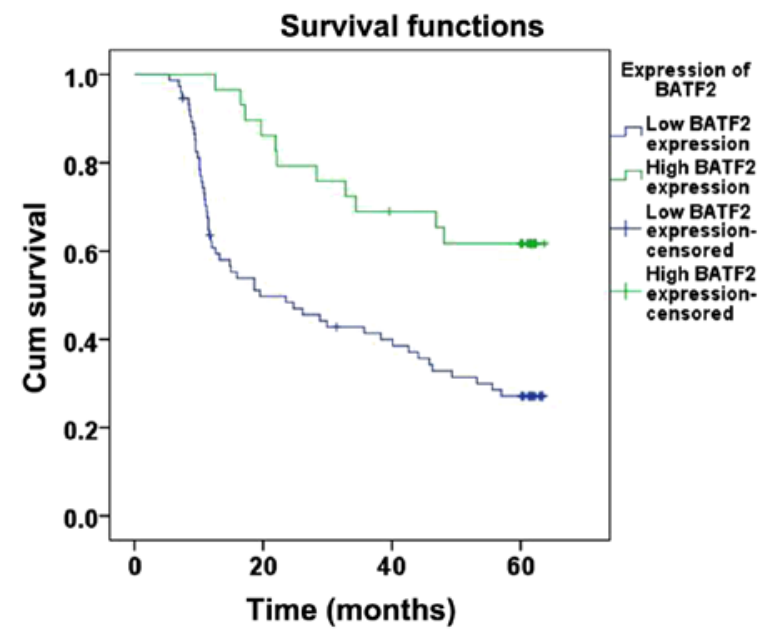

Figure 5. Kaplan-Meier analysis of overall survival of BATF2 in 104 patients with ESCC divided into groups with no or low expression (n=75) and moderate or high expression $(n=29)$. ESCC patients with moderate or high expression of BATF2 had a significantly prolonged survival rate compared to those with no or low expression of BATF2. Log-rank test, $\mathrm{P}<0.05$. BATF2, basic leucine zipper transcription factor, ATF-like 2; ESCC, esophageal squamous cell carcinoma.

motif, suggesting that it may be involved in transcriptional regulation (8,15-17). The gene encoding BATF2, which is expressed as multiple alternatively spliced isoforms, is located on the long arm of human chromosome 11 between 11q12 and 
Table II. Univariate and multivariate analyses of the overall survival rate in the 104 patients with ESCC.

\begin{tabular}{|c|c|c|c|}
\hline \multirow[b]{2}{*}{ Variables } & \multirow{2}{*}{$\begin{array}{l}\text { Univariate } \\
\text { analysis } \\
\text { P-value }\end{array}$} & \multicolumn{2}{|c|}{$\begin{array}{c}\text { Multivariate } \\
\text { analysis }\end{array}$} \\
\hline & & HR $(95 \% \mathrm{CI})$ & P-value \\
\hline \multicolumn{4}{|l|}{ Age (years) } \\
\hline$\leq 60.0$ & & 1 & \\
\hline$>60.0$ & 0.522 & $0.931(0.551-1.572)$ & 0.788 \\
\hline \multicolumn{4}{|l|}{ Gender } \\
\hline Male & & 1 & \\
\hline Female & 0.189 & $0.672(0.323-1.396)$ & 0.287 \\
\hline Location & 0.809 & & 0.903 \\
\hline $\mathrm{Ut}$ & & 1 & \\
\hline Mt & 0.694 & $1.132(0.605-2.118)$ & 0.699 \\
\hline $\mathrm{Lt}$ & 0.734 & $1.122(0.601-2.095)$ & 0.717 \\
\hline \multicolumn{4}{|l|}{$\begin{array}{l}\text { Pathological } \\
\text { grade }\end{array}$} \\
\hline Well & & 1 & \\
\hline Mod/poor & $0.016^{\mathrm{a}}$ & $1.864(1.072-3.240)$ & $0.027^{\mathrm{a}}$ \\
\hline \multicolumn{4}{|l|}{$\begin{array}{l}\text { Lymph node } \\
\text { metastasis }\end{array}$} \\
\hline Negative & & 1 & \\
\hline Positive & $<0.001^{\mathrm{a}}$ & $2.866(1.488-5.520)$ & $0.002^{\mathrm{a}}$ \\
\hline \multicolumn{4}{|l|}{ Clinical stage } \\
\hline IA-IIB & & 1 & \\
\hline IIIA-IV & $<0.001^{\mathrm{a}}$ & $4.028(2.060-7.875)$ & $<0.001^{\mathrm{a}}$ \\
\hline \multicolumn{4}{|l|}{$\begin{array}{l}\text { BATF2 } \\
\text { expression }\end{array}$} \\
\hline High & & 1 & \\
\hline Low or absent & $0.001^{\mathrm{a}}$ & $2.181(1.088-4.375)$ & $0.028^{\mathrm{a}}$ \\
\hline
\end{tabular}

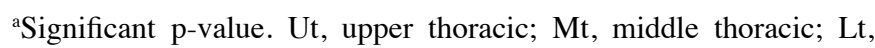
lower thoracic, ESCC, esophageal squamous cell carcinoma; HR, hazard ratio.

11q13. It consists of 3 exons, and translation starts in exon 1 and the stop codon is located in exon 3 (8). It was found that BATF2 is an important positive transcriptional regulator in the immune system and it is particularly important in cDC development, in $\mathrm{T}_{\mathrm{H}}$ cell function and in antibody production (18). Moreover, BATF2 is a melanoma differentiation-associated gene ( $m d a)$. As members of the mda family, mda-7 (19) and mda-9 (20) have been found to have an important role in tumor suppression (21), tumor migration and metabolism (22). Recently, it was found that decreased expression of BATF2 is associated with a poor prognosis in hepatocellular carcinoma, oral tongue squamous cell carcinoma, colorectal carcinomas and non-small cell lung cancer $(9,11,12,23)$, and a low level of BATF2 mRNA expression was found in CML patients (24). Furthermore, Wang et al found that BATF2 also can modulate epithelial-mesenchymal transition and lung adenocarcinoma metastasis (10). Steady-state expression of BATF2 has been observed in normal cells of diverse lineages, such as melanocytes, astrocytes, breast and prostate epithelial cells, and pancreatic mesothelial cells, yet expression was not detected in multiple cancer cell lines of the same tissue origin (8). However, there is no research study concerning the role of BATF2 in ESCC cell lines and the correlation between BATF2 expression and prognosis of ESCC.

In the present study, we revealed that the BATF2 expression level was downregulated at both the mRNA and protein levels in ESCC tissues and ESCC cell lines when compared with adjacent non-tumor tissues and a normal cell line as analyzed by qRT-PCR and western blot analysis. To the best of our knowledge, this is the first study to find that the expression of BATF2 is lower in ESCC than that in adjacent non-cancer tissues. Additionally, it was hypothesized by Ma et al that the loss of BATF2 may be a frequent event in human epithelial malignancies (11), and our findings may afford evidence to this hypothesis.

Immunohistochemical analysis indicated that the expression of BATF2 in ESCC tissues was related to tumor differentiation and lymph node metastasis. Given the association between BATF2 expression and tumor differentiation and lymph node metastasis by immunohistochemistry, we hypothesized that BATF2 plays an important role in tumor proliferation and metastasis. To address this issue, MTT and Transwell assays were performed. The result revealed that the BATF2-overexpression KYSE-410 cells had lower proliferation and invasion rates than these rates in the KYSE410 cells. A recent study also showed that the proliferation of A549 cells transfected with BATF2 was significantly inhibited as compared to the normal control group (9), which was consistent with our research using the ESCC cell line. Kaplan-Meier survival curves and the log-rank test survival analysis showed that the OS of patients with a low level of BATF2 was significantly reduced when compared with patients with a high level of BATF2. Cox multivariate analysis found that BATF2 protein expression is an independent prognostic predictor for ESCC after resection.

In summary, we found that BATF2 was significantly downregulated in ESCC tissues and the downregulation of BATF2 was correlated with a poor survival prognosis in ESCC patients. Moreover, expression of BATF2 in the ESCC tissues was related to the clinical properties of tumor differentiation and lymph node metastasis. In the ESCC cell lines, the expression of BATF2 was also decreased when compared with a normal esophageal epithelial cell line. In addition, in vitro studies revealed that overexpression of BATF2 reduced the proliferation and invasion ability of the human ESCC cell line KYSE-410. Yet, further research is required to elucidate the molecular mechanism of BATF2 in ESCC. Taken together, the results suggest that, as a tumor-suppressor, BATF2 may serve as a prognostic biomarker of ESCC and BATF2 may be a potential therapeutic target for ESCC treatment.

\section{Acknowledgements}

This study was funded by the National Nature Science Foundation of China (grant no. 81201890) and the Natural Science Foundation of Liaoning Province (grant no. 2013021002). 


\section{References}

1. Jemal A, Bray F, Center MM, Ferlay J, Ward E and Forman D: Global cancer statistics. CA Cancer J Clin 61: 69-90, 2011.

2. Hongo M, Nagasaki Y and Shoji T: Epidemiology of esophageal cancer: Orient to Occident. Effects of chronology, geography and ethnicity. J Gastroenterol Hepatol 24: 729-735, 2009.

3. Stoner GD and Gupta A: Etiology and chemoprevention of esophageal squamous cell carcinoma. Carcinogenesis 22: $1737-1746,2001$.

4. Lambert R and Hainaut P: The multidisciplinary management of gastrointestinal cancer. Epidemiology of oesophagogastric cancer. Best Pract Res Clin Gastroenterol 21: 921-945, 2007.

5. Thallinger CM, Kiesewetter B, Raderer M and Hejna M: Pre- and postoperative treatment modalities for esophageal squamous cell carcinoma. Anticancer Res 32: 4609-4627, 2012.

6. Kranzfelder M, Büchler P and Friess H: Surgery within multimodal therapy concepts for esophageal squamous cell carcinoma (ESCC): The MRI approach and review of the literature. Adv Med Sci 54: 158-169, 2009.

7. Kamangar F, Dores GM and Anderson WF: Patterns of cancer incidence, mortality, and prevalence across five continents: Defining priorities to reduce cancer disparities in different geographic regions of the world. J Clin Oncol 24: 2137-2150, 2006.

8. Su ZZ, Lee SG, Emdad L, Lebdeva IV, Gupta P, Valerie K, Sarkar D and Fisher PB: Cloning and characterization of SARI (suppressor of AP-1, regulated by IFN). Proc Natl Acad Sci USA 105: 20906-20911, 2008.

9. Zhou RJ, Shi Z, Zhou K, Wang HD, Zhang GQ, Li XT and Xu JP: Decreased SARI expression predicts poor prognosis of Chinese patients with non-small cell lung cancer. Int J Clin Exp Pathol 6 : 2056-2063, 2013.

10. Wang C, Su Y, Zhang L, Wang M, You J, Zhao X, Zhang Z, Liu J and Hao X: The function of SARI in modulating epithelial-mesenchymal transition and lung adenocarcinoma metastasis. PLoS One 7: e38046, 2012.

11. Ma H, Liang X, Chen Y, Pan K, Sun J, Wang H, Wang Q, Li Y, Zhao J, Li J, et al: Decreased expression of BATF2 is associated with a poor prognosis in hepatocellular carcinoma. Int $\mathbf{J}$ Cancer 128: 771-777, 2011.
12. Wen H, Chen Y, Hu Z, Mo Q, Tang J and Sun C: Decreased expression of BATF2 is significantly associated with poor prognosis in oral tongue squamous cell carcinoma. Oncol Rep 31: 169-174, 2014.

13. Edge SB and Compton CC: The American Joint Committee on Cancer: The 7th edition of the AJCC cancer staging manual and the future of TNM. Ann Surg Oncol 17: 1471-1474, 2010.

14. Schmittgen TD and Livak KJ: Analyzing real-time PCR data by the comparative C(T) method. Nat Protoc 3: 1101-1108, 2008.

15. Landschulz WH, Johnson PF and McKnight SL: The leucine zipper: A hypothetical structure common to a new class of DNA binding proteins. Science 240: 1759-1764, 1988.

16. Turner R and Tjian R: Leucine repeats and an adjacent DNA binding domain mediate the formation of functional $\mathrm{cFos}-\mathrm{cJun}$ heterodimers. Science 243: 1689-1694, 1989.

17. O'Shea EK, Rutkowski R, Stafford WF III and Kim PS: Preferential heterodimer formation by isolated leucine zippers from fos and jun. Science 245: 646-648, 1989.

18. Murphy TL, Tussiwand R and Murphy KM: Specificity through cooperation: BATF-IRF interactions control immune-regulatory networks. Nat Rev Immunol 13: 499-509, 2013.

19. Britschgi C and Fey MF: Tumor suppressor genes in myeloid differentiation and leukemogenesis. Future Oncol 5: 245-257, 2009.

20. Lin JJ, Jiang H and Fisher PB: Melanoma differentiation associated gene- 9 , mda- 9 , is a human gamma interferon responsive gene. Gene 207: 105-110, 1998

21. Oikawa K, Tanaka M, Itoh S, Takanashi M, Ozaki T, Muragaki Y and Kuroda M: A novel oncogenic pathway by TLS-CHOP involving repression of MDA-7/IL-24 expression. Br J Cancer 106: 1976-1979, 2012

22. Aissaoui H, Prévost C, Boucharaba A, Sanhadji K, Bordet JC, Négrier C and Boukerche H: MDA-9/syntenin is essential for factor VIIa-induced signaling, migration, and metastasis in melanoma cells. J Biol Chem 290: 3333-3348, 2015.

23. Liu ZB, Yang Y, Ye XG, Wang L, Tian PY and Zhang YY: Expression and significance of SARI and CCN1 in human colorectal carcinomas. Zhonghua Yi Xue Za Zhi 91: 2397-2401, 2011 (In Chinese).

24. Huang Q, Yang Y, Li X and Huang S: Transcription suppression of SARI (suppressor of AP-1, regulated by IFN) by BCR-ABL in human leukemia cells. Tumour Biol 32: 1191-1197, 2011. 